ISSN 1855-3966 (printed edn.), ISSN 1855-3974 (electronic edn.)

ARS MATHEMATICA CONTEMPORANEA 16 (2019) 25-38

https://doi.org/10.26493/1855-3974.1281.c7f

(Also available at http://amc-journal.eu)

\title{
On the $k$-metric dimension of metric spaces
}

\author{
Alan F. Beardon \\ University of Cambridge, Centre for Mathematical Sciences, \\ Wilberforce Road, Cambridge CB3 OWB, United Kingdom \\ Juan A. Rodríguez-Velázquez * \\ Universitat Rovira i Virgili, Departament d'Enginyeria Informàtica i Matemàtiques, \\ Av. Països Catalans 26, 43007 Tarragona, Spain
}

Received 10 January 2017, accepted 27 June 2018, published online 26 August 2018

\begin{abstract}
The metric dimension of a general metric space was defined in 1953, applied to the set of vertices of a graph metric in 1975, and developed further for metric spaces in 2013. It was then generalised in 2015 to the $k$-metric dimension of a graph for each positive integer $k$, where $k=1$ corresponds to the original definition. Here, we discuss the $k$-metric dimension of general metric spaces.
\end{abstract}

Keywords: Metric spaces, metric dimension, $k$-metric dimension.

Math. Subj. Class.: 54E35, 05C12

\section{Introduction}

The metric dimension of a general metric space was introduced in 1953 in [4, p. 95] but attracted little attention until, about twenty years later, it was applied to the distances between vertices of a graph $[12,14,15,18]$. Since then it has been frequently used in graph theory, chemistry, biology, robotics and many other disciplines. The theory was developed further in 2013 for general metric spaces [1]. More recently, the theory of metric dimension has been generalised, again in the context of graph theory, to the notion of a $k$-metric dimension, where $k$ is any positive integer, and where the case $k=1$ corresponds to the original theory $[7,8,9,10,11]$. Here we develop the idea of the $k$-metric dimension both in graph theory and in metric spaces. As the theory is trivial when the space has at most two points, we shall assume that any space we are considering has at least three points.

\footnotetext{
* This research was supported in part by the Spanish government under the grant MTM2016-78227-C2-1-P.

E-mail addresses: afb@dpmms.cam.ac.uk (Alan F. Beardon), juanalberto.rodriguez@urv.cat (Juan A. Rodríguez-Velázquez)
} 
Finally, whenever we discuss a connected graph $G$, we shall always consider the metric space $(X, d)$, where $X$ is the vertex set of $G$, and $d$ is the usual graph metric in which the distance between two vertices is the smallest number of edges that connect them.

Let $(X, d)$ be a metric space. If $X$ is a finite set, we denote its cardinality by $|X|$; if $X$ is an infinite set, we put $|X|=+\infty$. In fact, it is possible to develop the theory with $|X|$ any cardinal number, but we shall not do this. The distances from a point $x$ in $X$ to the points $a$ in a subset $A$ of $X$ are given by the function $a \mapsto d(x, a)$, and the subset $A$ is said to resolve $X$ if each point $x$ is uniquely determined by this function. Thus $A$ resolves $X$ if and only if $d(x, a)=d(y, a)$ for all $a$ in $A$ implies that $x=y$; informally, if an object in $X$ knows its distance from each point of $A$, then it knows exactly where it is located in $X$. The class $\mathcal{R}(X)$ of subsets of $X$ that resolve $X$ is non-empty since $X$ resolves $X$. The metric dimension $\operatorname{dim}(X)$ of $(X, d)$ is the minimum value of $|S|$ taken over all $S$ in $\mathcal{R}(X)$. The sets in $\mathcal{R}(X)$ are called the metric generators, or resolving subsets, of $X$, and $S$ is a metric basis of $X$ if $S \in \mathcal{R}(X)$ and $|S|=\operatorname{dim}(X)$. A metric generator of a metric space $(X, d)$ is, in effect, a global co-ordinate system on $X$. For example, if $\left(x_{1}, \ldots, x_{m}\right)$ is an ordered metric generator of $X$, then the map $\Delta: X \rightarrow \mathbb{R}^{m}$ given by

$$
\Delta(x)=\left(d\left(x, x_{1}\right), \ldots, d\left(x, x_{m}\right)\right)
$$

is injective (for this vector determines $x$ ), so that $\Delta$ is a bijection from $X$ to a subset of $\mathbb{R}^{m}$, and $X$ inherits its co-ordinates from this subset.

Now let $k$ be a positive integer, and $(X, d)$ a metric space. A subset $S$ of $X$ is a $k$-metric generator for $X$ (see [8]) if and only if any pair of points in $X$ is distinguished by at least $k$ elements of $S$ : that is, for any pair of distinct points $u$ and $v$ in $X$, there exist $k$ points $w_{1}, w_{2}, \ldots, w_{k}$ in $S$ such that

$$
d\left(u, w_{i}\right) \neq d\left(v, w_{i}\right), \quad i=1, \ldots, k
$$

A $k$-metric generator of minimum cardinality in $X$ is called a $k$-metric basis, and its cardinality, which is denoted by $\operatorname{dim}_{k}(X)$, is called the $k$-metric dimension of $X$. Let $\mathcal{R}_{k}(X)$ be the set of $k$-metric generators for $X$. Since $\mathcal{R}_{1}(X)=\mathcal{R}(X)$, we see that $\operatorname{dim}_{1}(X)=\operatorname{dim}(X)$. Also, as inf $\varnothing=+\infty$, this means that $\operatorname{dim}_{k}(X)=+\infty$ if and only if no finite subset of $X$ is a $k$-metric generator for $X$.

Given a metric space $(X, d)$, we define the dimension sequence of $X$ to be the sequence

$$
\left(\operatorname{dim}_{1}(X), \operatorname{dim}_{2}(X), \ldots, \operatorname{dim}_{k}(X), \ldots\right),
$$

and we address the following two problems.

- Can we find necessary and sufficient conditions for a sequence $\left(d_{1}, d_{2}, d_{3}, \ldots\right)$ to be the dimension sequence of some metric space?

- How does the dimension sequence of $(X, d)$ relate to the properties of $(X, d)$ ?

In Sections 2, 3 and 4 we provide some basic results on the $k$-metric dimension, and in Section 5 we calculate the dimension sequences of some metric spaces. We then apply these ideas to the join of two metric spaces, and to the Cayley graph of a finitely generated group. 


\section{Bisectors}

As shown in [1], the ideas about metric dimension are best described in terms of bisectors. For distinct $u$ and $v$ in $X$, the bisector $B(u \mid v)$ of $u$ and $v$ is given by

$$
B(u \mid v)=\{x \in X: d(x, u)=d(x, v)\} .
$$

The complement of $B(u \mid v)$ is denoted by $B^{c}(u \mid v)$; thus

$$
B^{c}(u \mid v)=\{x \in X: d(x, u) \neq d(x, v)\}
$$

and this contains both $u$ and $v$. Whenever we speak of a bisector $B$, we shall assume that it is some bisector $B(u \mid v)$, where $u \neq v$, so that its complement $B^{c}$ is not empty.

Let us now consider the $k$-metric dimension from the perspective of bisectors. A subset $A$ of $X$ fails to resolve $X$ if and only if there are distinct points $u$ and $v$ in $X$ such that $d(u, a)=d(v, a)$ for all $a$ in $A$. Thus $A$ resolves $X$ if and only if $A$ is not contained in any bisector or, equivalently, if and only if for every bisector $B$, we have $\left|B^{c} \cap A\right| \geq 1$. This leads to an alternative (but equivalent) definition of the metric dimension $\operatorname{dim}(X)$, namely

$$
\operatorname{dim}(X)=\inf \left\{|A|: A \subset X \text { and, for all bisectors } B,\left|B^{c} \cap A\right| \geq 1\right\} .
$$

Again, this infimum may be $+\infty$. The extension to the $k$-metric dimension $\operatorname{dim}_{k}(X)$ of $X$ is straightforward:

$$
\operatorname{dim}_{k}(X)=\inf \left\{|A|: A \subset X \text { and, for all bisectors } B,\left|B^{c} \cap A\right| \geq k\right\} .
$$

Note that if $X$ is a finite set then $\operatorname{dim}_{|X|+1}(X)=+\infty$.

Clearly, the values $\operatorname{dim}_{k}(X)$ depend only on the class $\mathcal{B}$ of bisectors in $X$; for example, $\operatorname{dim}_{1}(X)=1$ if and only if there is some point in $X$ that is not in any bisector. More generally, in all cases, $\operatorname{dim}_{k}(X) \geq k$, and equality holds here if and only if there are $k$ points of $X$ that do not lie in any bisector. For example, if $X$ is the real, closed interval $[0,1]$ with the Euclidean metric, then $\operatorname{dim}_{k}(X)=k$ for $k=1,2$. For a more general example of this type, let $X=\{\sqrt{p}: p$ a prime number $\}$ with the Euclidean metric $d$. If $p$, $q$ and $r$ are primes, with $p \neq q$, then $\sqrt{r} \in B(\sqrt{p} \mid \sqrt{q})$ implies $\sqrt{r}=\frac{1}{2}(\sqrt{p}+\sqrt{q})$; hence $4 r=p+q+2 \sqrt{p q}$. Since $\sqrt{p q}$ is irrational, this is false; hence every bisector is empty. It follows that $\operatorname{dim}_{k}(X)=k$ for $k=1,2, \ldots$; thus the dimension sequence of $(X, d)$ is $(1,2,3, \ldots)$.

\section{The monotonicity of dimensions}

Let $(X, d)$ be a metric space. Then, from (2.1), we have $\operatorname{dim}_{k}(X) \leq \operatorname{dim}_{k+1}(X)$, but we shall now establish the stronger inequality $\operatorname{dim}_{k}(X)+1 \leq \operatorname{dim}_{k+1}(X)$ (which is $\operatorname{dim}_{k}(X)<\operatorname{dim}_{k+1}(X)$ when the dimensions are finite, but not when they are $\left.+\infty\right)$. This inequality is known for graphs; see $[8,10])$ where it is an important tool.

Theorem 3.1. Let $(X, d)$ be a metric space. Then, for $k=1,2, \ldots$,

(i) if $\operatorname{dim}_{k}(X)<+\infty$ then $\operatorname{dim}_{k}(X)<\operatorname{dim}_{k+1}(X)$;

(ii) if $\operatorname{dim}_{k}(X)=+\infty$ then $\operatorname{dim}_{k+1}(X)=+\infty$.

In particular, $\operatorname{dim}_{k}(X)+1 \geq \operatorname{dim}_{1}(X)+k$. 
Proof. First, (ii) follows immediately from (2.1). Next, (i) is true if $\operatorname{dim}_{k+1}(X)=+\infty$, so we may assume that $\operatorname{dim}_{k+1}(X)=p<+\infty$. Thus there is a subset $\left\{x_{1}, \ldots, x_{p}\right\}$ (with the $x_{i}$ distinct) of $X$ such that for every bisector $B,\left|B^{c} \cap\left\{x_{1}, \ldots, x_{p}\right\}\right| \geq k+1$. As $k \geq 1$ we see that $p \geq 2$. Clearly, $\left|B^{c} \cap\left\{x_{1}, \ldots, x_{p-1}\right\}\right| \geq k$ for every bisector $B$; hence $\operatorname{dim}_{k}(X) \leq p-1<\operatorname{dim}_{k+1}(X)$. The last inequality follows by induction.

\section{The 1-metric dimension}

Theorem 3.1 shows that if $+\infty$ occurs as a term in the dimension sequence of $(X, d)$, then all subsequent terms are also $+\infty$. Thus $\operatorname{dim}_{1}(X)=+\infty$ if and only if $(X, d)$ has dimension sequence $(+\infty,+\infty,+\infty, \ldots)$. The next result shows when this is so.

Theorem 4.1. Let $(X, d)$ be a metric space. Then $\operatorname{dim}_{1}(X)=+\infty$ if and only if every finite subset of $X$ lies in some bisector. In particular, if $X$ is the union of an increasing sequence of bisectors, then $\operatorname{dim}_{1}(X)=+\infty$.

Proof. First, the definition of $\operatorname{dim}(X)$ implies that $\operatorname{dim}_{1}(X)=+\infty$ if and only if every finite subset of $X$ lies in some bisector. The second statement holds because if $X=$ $\cup_{n} B_{n}$, where $B_{1}, B_{2}, \ldots$ is an increasing sequence of bisectors, then, given any finite subset $\left\{x_{1}, \ldots, x_{m}\right\}$ of $X$, each $x_{j}$ lies in some $B_{i_{j}}$, and $\left\{x_{1}, \ldots, x_{m}\right\} \subset B_{r}$, where $r=\max \left\{i_{1}, \ldots, i_{m}\right\}$.

What can be said if $\operatorname{dim}_{1}(X)<+\infty$ ? It seems that we can obtain very little information from the single assumption that $\operatorname{dim}_{1}(X)<+\infty$; for example, for each $r \geq 0$ choose a point $x_{r}$ in $\mathbb{R}^{n}$ with $\left\|x_{r}\right\|=r$, and let $X=\left\{x_{r}: r \geq 0\right\}$. Then $\{0\}$ is a 1-metric basis for $X$, and $\operatorname{dim}_{1}(X)=1$ but we can say almost nothing about the topological structure of $X$. However, we can say more if we know that $X$ is compact.

Theorem 4.2. Let $(X, d)$ be a compact metric space with $\operatorname{dim}_{1}(X)=m<+\infty$. Then $(X, d)$ is homeomorphic to a compact subset of $\mathbb{R}^{m}$.

Proof. Suppose that $X$ is compact, and that $\operatorname{dim}_{1}(X)=m<+\infty$. Then there is a 1metric basis $\left\{x_{1}, \ldots, x_{m}\right\}$, and the corresponding bijection $\Delta$ in (1.1) that maps $X$ onto some subset of $\mathbb{R}^{m}$. Now $\Delta$ is continuous on $X$ since

$$
|\Delta(x)-\Delta(y)| \leq \sum_{j=1}^{m}\left|d\left(x, x_{j}\right)-d\left(y, x_{j}\right)\right| \leq \operatorname{md}(x, y) .
$$

As $\Delta$ is a continuous, injective map from a compact space to the Hausdorff space $\mathbb{R}^{m}$ it follows (by a well known result in topology) that it is a homeomorphism.

This result is related to the following result in [1] (see also [16]).

Theorem 4.3. If $(X, d)$ is a compact, connected metric space with $\operatorname{dim}_{1}(X)=1$ then $X$ is homeomorphic to $[0,1]$.

The compactness is essential here as there is an example in [1] of a connected, but not arcwise connected, metric space $X$ with $\operatorname{dim}_{1}(X)=1$. As $X$ is not arcwise connected, it is not homeomorphic to [0,1]. It is conjectured in [1] that if $X$ is arcwise connected, and $\operatorname{dim}_{1}(X)=1$ then $X$ is a Jordan arc (this means that $X$ is homeomorphic to one of the real intervals $[0,1]$ and $[0,+\infty)$ ), and we can now show that this is so. 
Theorem 4.4. If $X$ is an arcwise connected metric space with $\operatorname{dim}_{1}(X)=1$, then $X$ is a Jordan arc.

Proof. As $\operatorname{dim}_{1}(X)=1$, there is a metric basis, say $\left\{x_{0}\right\}$ for $X$, and every point $x$ of $X$ is uniquely determined by its distance $d\left(x, x_{0}\right)$ from $x_{0}$. Consider the map $\Delta: x \mapsto d\left(x, x_{0}\right)$ of $X$ into $[0,+\infty)$. This map is (uniformly) continuous because

$$
|\Delta(x)-\Delta(y)|=\left|d\left(x, x_{0}\right)-d\left(y, x_{0}\right)\right| \leq d(x, y),
$$

and as $X$ is arcwise connected (and therefore connected), so $\Delta(X)$ is connected. This means that $\Delta$ is an interval of the form $[0, a]$, where $a>0$, or $[0, b)$, where $0<b \leq+\infty$.

Let us consider the case when $\Delta(X)=[0, a]$. As $\Delta$ is injective, we see that for every $r$ in the interval $[0, a]$ there is some unique $x_{r}$ in $X$ with $d\left(x_{r}, x_{0}\right)=r$. Thus $X=\left\{x_{r}\right.$ : $0 \leq r \leq a\}$. However, as $X$ is arcwise connected, there is a curve, say $\gamma:[0,1] \rightarrow X$ with $\gamma(0)=x_{0}$ and $\gamma(1)=x_{a}$. Now as $\gamma$ is continuous, the set $\left\{d\left(\gamma(t), x_{0}\right): t \in[0,1]\right\}$ must contain every real number in the interval $[0, a]$, and it cannot contain any other numbers; thus $X=\gamma([0,1])$. Now $\gamma([0,1])$ is compact for it is the continuous image of the compact interval $[0,1]$; thus $X$ is compact and so, by Theorem $4.3, X$ is a Jordan arc.

The argument in the case when $\Delta(X)=[0, b)$ is similar. Indeed, the argument above holds for every $a$ with $0<a<b$, and it is easy to see that this implies that $\Delta$ is a homeomorphism from $X$ to $[0, b)$.

\section{Some examples}

In order to calculate the $k$-metric dimension of a metric space we need to understand the geometric structure of its bisectors, and we now illustrate this with several examples. In order to maintain the flow of ideas, the details of these examples will be given later.

Example 5.1. Let $(X, d)$ be any one of the Euclidean, spherical and hyperbolic spaces $\mathbb{R}^{n}$, $\mathbb{S}^{n}$ and $\mathbb{H}^{n}$, respectively, each with the standard metric of constant curvature 0,1 and -1 , respectively. The bisectors are well understood in these spaces, and we shall show that any non-empty open subset of $X$ has $k$-metric dimension $n+k$. In particular, each of these spaces has dimension sequence $(n+1, n+2, n+3, \ldots)$. See [1, 13] for the 1-metric dimensions of these spaces.

Example 5.2. Let $X$ be any finite set with the discrete metric $d$ (equivalently, $X$ is the vertex set of a complete, finite graph). For distinct $u$ and $v$ in $X$ we have $B(u \mid v)=$ $X \backslash\{u, v\}$, so that for any subset $S$ of $X$, we have $B(u \mid v)^{c} \cap S=\{u, v\} \cap S$. Thus if $\left|S \cap B^{c}\right| \geq 1$ for all bisectors $B$, then $S$ can omit at most one point of $X$. We conclude that $\operatorname{dim}_{1}(X)=|X|-1$. If $\left|B^{c} \cap S\right| \geq 2$ for all bisectors $B$ then $S=X$, and $\operatorname{dim}_{2}(X)=|X|$. We conclude that $(X, d)$ has dimension sequence $(|X|-1,|X|,+\infty,+\infty, \ldots)$.

Example 5.3. Let $X$ be the real interval $[0,1]$, with the Euclidean metric. Then $B$ is a bisectors if and only if $B=\{x\}$ for some $x$ in $(0,1)$. Thus $\{0\}$ is a 1 -metric basis, and $\{0,1\}$ is a 2 -metric basis, of $[0,1]$. We leave the reader to show that if $k \geq$ 3 then $\left\{0, \frac{1}{k}, \frac{2}{k}, \ldots, \frac{k-1}{k}, 1\right\}$ is a $k$-metric basis, so that $[0,1]$ has dimension sequence $(1,2,4,5,6, \ldots)$. A similar argument shows that $[0,+\infty)$ has dimension sequence $(1,3,4$, $5, \ldots)$, and that $(-\infty,+\infty)$, which is $\mathbb{R}$, has dimension sequence $(2,3,4, \ldots)$.

Example 5.4. The Petersen graph, which is illustrated in Figure 1, has dimension sequence $(3,4,7,8,9,10,+\infty, \ldots)$. The (finite) values $\operatorname{dim}_{k}(X)$ for $k=1, \ldots, 6$ come from a computer search, and as $\operatorname{dim}_{6}(X)=10=|X|$, we have $\operatorname{dim}_{7}(X)=+\infty$. 


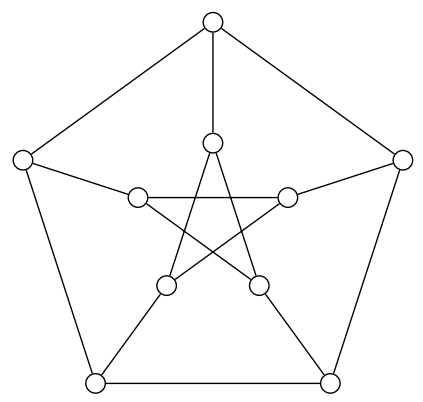

Figure 1: The Petersen graph.

Example 5.5. Let $G$ be a group with a given set of generators, let $V$ be the vertex set of the associated Cayley graph of $G$, and let $d$ be its graph metric.

(i) If $G$ is an infinite cyclic group then $(V, d)$ has dimension sequence $(2,3,4, \ldots)$.

(ii) If $G$ is a free group on $p$ generators, where $p \geq 2$, then $(V, d)$ has dimension sequence $(+\infty,+\infty,+\infty, \ldots)$.

(iii) Let $G$ be an abelian group on $p$ generators, where $p \geq 2$, and where each generator has infinite order. Then $(V, d)$ has dimension sequence $(+\infty,+\infty,+\infty, \ldots)$.

\section{Three geometries of constant curvature}

In this section we give the details of Example 5.1. It is shown in [1] that if $U$ is any non-empty, open subset of any one of the three classical geometries $\mathbb{R}^{n}, \mathbb{S}^{n}$ and $\mathbb{H}^{n}$, then $\operatorname{dim}_{1}(U)=n+1$. Here we show that if $X$ is any of these spaces then $\operatorname{dim}_{k}(X)=n+k$ for $k=1,2, \ldots$. The same result holds for non-empty open subsets of these spaces, and we leave the reader to make the appropriate changes to the proofs.

The proof that $\operatorname{dim}_{k}(X)=n+k$ when $X$ is one of the three geometries $\mathbb{R}^{n}, \mathbb{S}^{n}$ and $\mathbb{H}^{n}$, is largely independent of the choice of $X$, and depends only on the nature of the bisectors in these geometries. Each of these three geometries has the following properties:

(P1) $\operatorname{dim}_{1}(X)=n+1$;

(P2) there exists $x_{1}, x_{2}, \ldots$ in $X$ such that if $j_{1}<j_{2}<\cdots<j_{n}$ then $\left\{x_{j_{1}}, \ldots, x_{j_{n}}\right\}$ lies on a unique bisector $B$, and no other $x_{i}$ lies on $B$.

Now (P1) and (P2) imply that $\operatorname{dim}_{k}(X)=n+k$ for $k=1,2, \ldots$. Indeed, (P2) implies that for any bisector $B,\left|B \cap\left\{x_{1}, \ldots, x_{n+k}\right\}\right| \leq n$, so that $\left|B^{c} \cap\left\{x_{1}, \ldots, x_{n+k}\right\}\right| \geq k$. This implies that $\operatorname{dim}_{k}(X) \leq n+k$. However, (P1) and Theorem 3.1 show that $\operatorname{dim}_{k}(X) \geq$ $n+k$. Since we know that each of $\mathbb{R}^{n}, \mathbb{S}^{n}$ and $\mathbb{H}^{n}$ has the property (P1), it remains to show that they have the property (P2), and this depends on the nature of the bisectors in these geometries. We consider each in turn.

\subsection{Euclidean space $\mathbb{R}^{n}$}

Each bisector in $\mathbb{R}^{n}$ is a hyperplane (that is, the translation of an $(n-1)$-dimensional subspace of $\mathbb{R}^{n}$ ), and each hyperplane is a bisector. Any set of $n$ points lies on a bisector, 
and there exists sets of $n+1$ points that do not lie on any single bisector. The appropriate geometry here is the affine geometry of $\mathbb{R}^{n}$, but we shall take a more informal view. First, we choose $n$ points $x_{1}, \ldots, x_{n}$ that lie on a unique hyperplane $H$. Next, we select a point $x_{n+1}$ not on $H$. Then any $n$ points chosen from $\left\{x_{1}, \ldots, x_{n+1}\right\}$ lie on some hyperplane $H^{\prime}$, and the remaining point does not lie on $H^{\prime}$. Now suppose that we have constructed the set $\left\{x_{1}, \ldots, x_{n+p}\right\}$ with the property that any set of $n$ points chosen from this lie on a unique hyperplane, say $H_{\alpha}$, and that no other $x_{i}$ lies on $H_{\alpha}$. Then we can choose a point $x_{n+p+1}$ that is not on any of the $\left(\begin{array}{c}n+p \\ n\end{array}\right)$ hyperplanes $H_{\alpha}$, and it is then easy to check that the sequence $x_{1}, x_{2}, \ldots$ has the property (P2).

Although we have not used it, we mention that there is a formula for the $n$-dimensional volume $V$ of the Euclidean simplex whose vertices are the $n+1$ points $x_{1}, \ldots, x_{n+1}$ in $\mathbb{R}^{n}$, namely

$$
V^{2}=\frac{(-1)^{n+1}}{2^{n}(n !)^{2}} \Delta,
$$

where $\Delta$ is the Cayley-Menger determinant given by

$$
\Delta=\left|\begin{array}{cccc}
0 & 1 & \cdots & 1 \\
1 & d_{1,1}^{2} & \cdots & d_{1, n+1}^{2} \\
\vdots & \vdots & \ddots & \vdots \\
1 & d_{n+1,1}^{2} & \cdots & d_{n+1, n+1}^{2}
\end{array}\right|
$$

and $d_{i, j}=\left\|x_{i}-x_{j}\right\|$. As $V=0$ precisely when the points $x_{j}$ lie on a hyperplane, we see that this condition could be used to provide an algebraic background to the discussion above. For more details, see [3, 4] and [5]. We also mention that there are versions of the Cayley-Menger determinant that are applicable to spherical, and to hyperbolic, spaces.

\subsection{Spherical space $\mathbb{S}^{n}$}

Spherical space $\left(\mathbb{S}^{n}, d\right)$ is the space $\left\{x \in \mathbb{R}^{n+1}:\|x\|=1\right\}$ with the path metric $d$ induced on $\mathbb{S}^{n}$ by the Euclidean metric on $\mathbb{R}^{n+1}$. Explicitly, $\cos d(x, y)=x \cdot y$, where $x \cdot y$ is the usual scalar product in $\mathbb{R}^{n+1}$. If $u$ and $v$ are distinct points of $\mathbb{S}^{n}$, we let $B^{\mathcal{E}}(u \mid v)$ be the Euclidean bisector (in $\mathbb{R}^{n+1}$ ) of $u$ and $v$, and $B^{\mathcal{S}}(u \mid v)$ the spherical bisector in the space $\left(\mathbb{S}^{n}, d\right)$. Then $B^{\mathcal{E}}(u \mid v)$ is a hyperplane that passes through the origin in $\mathbb{R}^{n+1}$, and

$$
B^{\mathcal{S}}(u \mid v)=\mathbb{S}^{n} \cap B^{\mathcal{E}}(u \mid v) .
$$

The bisectors $B^{\mathcal{S}}(u \mid v)$ are the great circles (of the appropriate dimension) on $\mathbb{S}^{n}$.

The equation (6.1) implies that the $k$-metric dimension of the spherical spaces is the same as for Euclidean spaces. Indeed, our proof for Euclidean spaces depended on constructing a sequence $x_{1}, x_{2}, \ldots$ with the property (P2), and it is clear that this construction could be carried out in such a way that each $x_{j}$ lies on $\mathbb{S}^{n}$.

\subsection{Hyperbolic space $\mathbb{H}^{n}$}

Our model of hyperbolic $n$-dimensional space is Poincare's half-space model

$$
\mathbb{H}^{n}=\left\{\left(x_{1}, \ldots, x_{n}\right) \in \mathbb{R}^{n}: x_{n}>0\right\}
$$

equipped with the hyperbolic distance $d$ which is derived from Riemannian metric $|d x| / x_{n}$. For more details, see for example, [2, 17]. Our argument for $\mathbb{H}^{n}$ is essentially the same as 
for $\mathbb{R}^{n}$ and $\mathbb{S}^{n}$ because if $u$ and $v$ are distinct points in $\mathbb{H}^{n}$, then the hyperbolic bisector $B(u \mid v)$ is the set $S \cap \mathbb{H}^{n}$, where $S$ is some Euclidean sphere whose centre lies on the hyperplane $x_{n}=0$. We omit the details.

\section{The metric dimensions of graphs}

The vertex set $V$ of a graph $G$ supports a natural graph metric $d$, where $d(u, v)$ is the smallest number of edges that can be used to join $u$ to $v$. Some basic results on the $k$ metric dimension of a graph have recently been obtained in $[7,8,9,10,11]$. Moreover, it was shown in [19] that the problem of computing the $k$-metric dimension of a graph is NP-hard. A natural problem in the study of the $k$-metric dimension of a metric space $(X, d)$ consists of finding the largest integer $k$ such that there exists a $k$-metric generator for $X$. For instance, for the graph shown in Figure 2 the maximum value of $k$ is four. It was shown in $[7,10]$ that for any graph of order $n$ this problem has time complexity of order $O\left(n^{3}\right)$. If we consider the discrete metric space $\left(X, d_{0}\right)$ (equivalently, a compete graph), then $\operatorname{dim}_{1}(X)=|X|-1$ and $\operatorname{dim}_{2}(X)=|X|$. Furthermore, for $k \geq 3$ there are no $k$-metric generators for $X$. In general, for any metric space $(X, d)$, the whole space $X$ is a 2-metric generator, as two vertices are distinguished by themselves. As we have already seen, there are metric spaces, like the Euclidean space $\mathbb{R}^{n}$, where for any positive integer $k$, there exist at least one $k$-metric generator.

We shall now discuss the dimension sequences of the simplest connected graphs, that is paths and cycles (and we omit the elementary details).

A finite path $P_{n}$ is a graph with vertices $v_{1}, \ldots, v_{n}$, edges $\left\{v_{1}, v_{2}\right\}, \ldots,\left\{v_{n-1}, v_{n}\right\}$, and bisectors $\left\{v_{2}\right\}, \ldots,\left\{v_{n-1}\right\}$. We leave the reader to show that $P_{n}$ has dimension sequence

$$
\begin{cases}(1,2,+\infty, \ldots) & \text { if } n=2,3 \\ (1,2,4,5, \ldots, n,+\infty, \ldots) & \text { if } n \geq 4\end{cases}
$$

A semi-infinite path $P_{\mathbb{N}}$ is a graph with vertices $v_{1}, v_{2} \ldots$, edges $\left\{v_{1}, v_{2}\right\},\left\{v_{2}, v_{3}\right\}, \ldots$, and bisectors $\left\{v_{2}\right\}, \ldots$ Thus $P_{\mathbb{N}}$ has dimension sequence $(1,3,4,5, \ldots)$. A doubly-infinite path $P_{\mathbb{Z}}$ is the graph with vertices $\ldots, v_{-1}, v_{0}, v_{1}, \ldots$, edges $\ldots,\left\{v_{-1}, v_{0}\right\},\left\{v_{0}, v_{1}\right\}, \ldots$, and bisectors $\ldots,\left\{v_{-1}\right\},\left\{v_{0}\right\},\left\{v_{1}\right\}, \ldots$ Thus $P_{\mathbb{Z}}$ has dimension sequence $(2,3,4,5, \ldots)$. We note that a graph $G$ has 1 -metric dimension 1 if and only it is $P_{n}$ or $P_{\mathbb{N}}[6,14]$. This, together with the results just stated, show that if $G$ is a graph of order two or more, and $k \geq 2$, then $\operatorname{dim}_{k}(G)=k$ if and only if $G$ is $P_{n}$ and $k=2$ (see also [8]).

We now consider cycles. A cycle $C_{n}$ is a graph with vertices $v_{1}, \ldots, v_{n}$, and edges $\left\{v_{1}, v_{2}\right\}, \ldots,\left\{v_{n-1}, v_{n}\right\},\left\{v_{n}, v_{1}\right\}$. We must distinguish between the cases where $n$ is even, and where $n$ is odd (which is the easier of the two cases) and, as typical examples, we mention that $C_{7}$ has dimension sequence $(2,3, \ldots, 7,+\infty, \ldots)$, and $C_{8}$ has dimension sequence $(2,3,4,6,7,8,+\infty, \ldots)$. Suppose that $n$ is odd; then the bisectors are the singletons $\{v\}$. Thus if $S$ is a set of $k+1$ vertices, where $k+1 \leq n$, then $\left|B^{c} \cap S\right| \geq k$ for every bisector $B$. Thus if $n$ is odd, then $\operatorname{dim}_{k}\left(C_{n}\right)=k+1$, and $C_{n}$ has dimension sequence $(2,3, \ldots, n,+\infty, \ldots)$.

We now show that $C_{2 q}$ has dimension sequence

$$
(2,3, \ldots, q, q+2, q+3, \ldots, q+q,+\infty, \ldots) .
$$

To see this, label the vertices as $v_{j}$, where $j \in \mathbb{Z}$, and where $v_{i}=v_{j}$ if and only if $i \equiv j$ $(\bmod n)$. The vertices $v_{i}$ and $v_{j}$ are antipodal vertices if and only if $i-j \equiv q(\bmod 2 q)$; 
thus $v_{j}$ and $v_{j+q}$ are antipodal vertices. The class of bisectors is the class of sets $\left\{v, v^{*}\right\}$, where $v$ is a vertex, and $v^{*}$ is the vertex that is antipodal to $v$. For $k=1, \ldots, q-1$ we can take a set of $k+1$ points, no two of which are antipodal, as a $k$-metric basis, so that $\operatorname{dim}_{k}\left(C_{2 q}\right)=k+1$ for $k=1, \ldots, q-1$. To find $\operatorname{dim}_{q}\left(C_{2 q}\right)$, we need to take (for a $q$-metric basis) a set $S$ which contains two pairs of antipodal points, and one more point from each pair of the remining antipodal pairs. We leave the details to the reader.

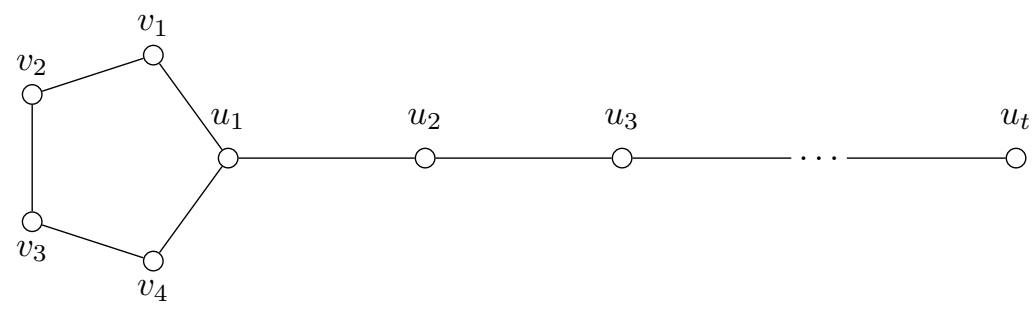

Figure 2: For $k \in\{1,2,3,4\}, \operatorname{dim}_{k}(G)=k+1$.

As an example which joins a path to a cycle, consider the graph $G$ illustrated in Figure 2 which is obtained from the cycle graph $C_{5}$ and the path $P_{t}$, by identifying one of the vertices of the cycle, say $u_{1}$, and one of the end vertices of $P_{t}$. Let $S_{1}=\left\{v_{1}, v_{2}\right\}, S_{2}=$ $\left\{v_{1}, v_{2}, u_{t}\right\}, S_{3}=\left\{v_{1}, v_{2}, v_{3}, u_{t}\right\}$ and $S_{4}=\left\{v_{1}, v_{2}, v_{3}, v_{4}, u_{t}\right\}$. Then, for $k=1,2,3,4$, the set $S_{k}$ is $k$-metric basis of $G$.

The following lemma is useful when discussing examples in graph theory.

Lemma 7.1. Suppose that a graph $G$ does not have any cycles of odd length. Then $B(u \mid v)=\varnothing$ when $d(u, v)$ is odd.

The proof is trivial for if $x \in B(u \mid v)$ then there is a cycle of odd length (from $u$ to $x$, then to $v$, and then back to $u$ ). This lemma applies, for example, to the usual grid (or graph) in $\mathbb{R}^{n}$ whose vertex set is $\mathbb{Z}^{n}$. A bipartite graph is a graph $G$ whose vertex set $V$ splits into complementary sets $V_{1}$ and $V_{2}$ such that each of the edges of $G$ join a point of $V_{1}$ to a point of $V_{2}$. As a graph is bipartite if and only if it has no cycles of an odd length, this lemma is about bipartite graphs.

Example 7.2. Let us now consider a graph $G$ that is an infinite tree in which every vertex has degree at least three. Now let $v$ be any vertex, select three edges from $v$, say $\{v, a\}$, $\{v, b\}$ and $\{v, c\}$. As $G$ is a tree, if we remove one edge the remaining graph is disconnected. Now let $G_{c}$ be the subgraph of $G$ that would be the component containing $c$ if we were to remove the edge $\{v, c\}$ from $G$. It is clear that if $u$ is a vertex in $G_{c}$, then $d(a, u)=d(b, u)$ since any path from $a$ (or $b$ ) to $u$ must pass through the edge $\{v, c\}$. We conclude that $G_{c} \subset B(a \mid b)$. It is now clear from Theorem 4.1 that $G$ has dimension sequence $(+\infty,+\infty, \ldots)$.

For the rest of this section we shall consider the Cayley graph of a group with a given set of generators as a metric space. Let $G$ be a group and let $G_{0}$ a set of generators of $G$. We shall always assume that if $g \in G_{0}$ then $g^{-1} \in G_{0}$ also. Then the Cayley graph of the pair $\left(G, G_{0}\right)$ is a graph whose vertex set is $G$, and such that the pair $\left(g_{1}, g_{2}\right)$ is an edge if and only if $g_{2}=g_{0} g_{1}$ for some $g_{0}$ in $G_{0}$. Thus, for example, $P_{\mathbb{Z}}$ is the Cayley graph of an infinite cyclic group (on one generator), and $C_{n}$ is the Cayley graph of an finite 
cyclic group (on one generator). We shall always assume that the set $G_{0}$ of generators of $G$ is finite; then the Cayley graph is locally finite (that is, each vertex is the endpoint of only finitely many edges). Note also that if a generator $g_{0}$ has order two then $g_{0}^{-1}=g_{0}$ so this only provides one edge (not two edges) from each vertex. The following result, which characterises Cayley graphs within the class of all graphs, is well known.

Theorem 7.3. A graph is a Cayley graph of a group $G$ if and only if it admits a simply transitive action of $G$ by graph automorphisms.

Theorem 7.3 suggests that if we use the homogeneity implied by this result there is a reasonable chance of finding the dimension sequence of a Cayley graph. However, for a graph that is not the Cayley graph of a group, it seems that we are reduced to finding its metric dimensions by a case by case analysis.

We shall now verify the claims made in Example 5.5. First, suppose that $G$ is a free group on $p$ generators. Then the Cayley graph of $G$ is a tree in which every vertex has degree $2 p$; thus, using Example 7.2, we see that $G$ has dimension sequence $(+\infty,+\infty$, $+\infty, \ldots)$.

Next, we consider an abelian group $G$ on two generators of infinite order (the proof for $p$ generators is entirely similar). The Cayley graph of $G$ has $\mathbb{Z}^{2}$ as its vertex set and (if we identify the lattice point $(m, n)$ with the Gaussian integer $m+i n)$ edges $\{m+i n, m+1+$ $i n\}$ and $\{m+i n, m+i(n+1)\}$, where $m, n \in \mathbb{Z}$. It is (geometrically) clear that for any $m \in \mathbb{Z}$ we have, with $\zeta=m+i m$,

$$
B(\zeta+1 \mid \zeta+i) \supset\{p+i q: p \geq m+1, q \geq m+1\} .
$$

It now follows from Theorem 4.1 (by taking $|m|$ large and $m$ negative) that $G$ has dimension sequence $(+\infty,+\infty, \ldots)$.

In contrast to Example 5.5 we have the following result for the infinite dihedral group whose Cayley graph is an infinite ladder; for example we can take the group generated by the two Euclidean isometries which, in complex terms, are $z \mapsto z+1$ and $z \mapsto \bar{z}$.

Theorem 7.4. The infinite dihedral group has dimension sequence $(3,4,6,8, \ldots)$.

Proof. We may assume that (in complex terms) the vertices of the ladder graph are the points $m+i n$, where $m \in \mathbb{Z}$ and $n=0,1$. The key to computing the metric dimensions of the ladder graph is the observation that

$$
B(0 \mid 1+i)=\{1,2,3, \ldots\} \cup\{i, i-1, i-2, \ldots\} .
$$

Of course, similar bisectors arise at other pairs of similarly located points; equivalently, each automorphism of the graph maps a bisector to a bisector. All other bisectors are either empty or of cardinality two. We claim that $\{0,1, i\}$ is a 1 -metric basis for the graph so that $\operatorname{dim}_{1}(G)=3$. Next, it is easy to see that $\{0,1, i, 1+i\}$ is a 2 -metric basis for $X$ so that $\operatorname{dim}_{2}(X)=4$. The set $\{0,1,2, i, 1+i, 2+i\}$ is a 3 -metric basis so that $\operatorname{dim}_{3}(X)=6$. We leave the details, and the remainder of the proof to the reader.

\section{The join of metric spaces}

The $k$-metric dimension of the join $G_{1}+G_{2}$ of two finite graphs $G_{1}$ and $G_{2}$ was studied in [7]. Let us briefly recall the notion of the join of two graphs $G_{1}$ and $G_{2}$ with disjoint 
vertex sets $V_{1}$ and $V_{2}$, respectively. The join $G_{1}+G_{2}$ of $G_{1}$ and $G_{2}$ is the graph whose vertex set is $V_{1} \cup V_{2}$, and whose edges are the edges in $G_{1}$, the edges in $G_{2}$, together with all edges obtained by joining each point in $V_{1}$ to each point in $V_{2}$. Let $d_{1}, d_{2}$ and $d$ be the graph metrics of $G_{1}, G_{2}$ and $G_{1}+G_{2}$, respectively; then

$$
d(u, v)= \begin{cases}\min \left\{d_{1}(u, v), 2\right\} & \text { if } u, v \in V_{1} \\ \min \left\{d_{2}(u, v), 2\right\} & \text { if } u, v \in V_{2} \\ 1 & \text { if } u \in V_{i}, v \in V_{j}, \text { where } i \neq j\end{cases}
$$

because if $u, v \in V_{1}$, say, then for $w$ in $V_{2}$, we have $d(u, v) \leq d(u, w)+d(w, v)=2$.

The join of two metric spaces is defined in a similar way, but before we do this we recall that if $(X, d)$ is a metric space, and $t>0$, then $d^{t}$, defined by

$$
d^{t}(x, y)=\min \{d(x, y), 2 t\}
$$

is a metric on $X$. If $d(x, y)<2 t$ then $d^{t}(x, y)=d(x, y)$, so that the $d^{t}$-metric topology coincides with the $d$-metric topology on $X$. As the metric $d^{t}$ will appear in our definition of the join, we first show how the metric dimension of a single metric space varies when we distort the metric from $d$ to $d^{t}$ as above. From now on, the $k$-metric dimension of $\left(X, d^{t}\right)$ will be denoted by $\operatorname{dim}_{k}^{t}(X)$.

Theorem 8.1. Let $(X, d)$ be a metric space, and $k$ a positive integer, and suppose that $0<s<t$. Then $\operatorname{dim}_{k}^{s}(X) \geq \operatorname{dim}_{k}^{t}(X) \geq \operatorname{dim}_{k}(X)$. However, it can happen that

$$
\lim _{t \rightarrow+\infty} \operatorname{dim}_{k}^{t}(X)>\operatorname{dim}_{k}(X)
$$

The join of two metric spaces is defined in a similar way to the join of two graphs, and to motivate this, suppose that $(X, d)$ is a metric space, and that $X_{1}$ and $X_{2}$ are bounded subsets $X$ whose distance apart is very large compared with their diameters. Then, in some sense, we can approximate the metric space $\left(X_{1} \cup X_{2}, d\right)$ by replacing all values $d\left(x_{1}, x_{2}\right)$, where $x_{j} \in X_{j}$, by $t$, where $t$ is some sort of average of the values $d\left(x_{1}, x_{2}\right)$. We shall now define the join, so suppose that $\left(X_{1}, d_{1}\right)$ and $\left(X_{2}, d_{2}\right)$ are metric spaces, with $X_{1} \cap X_{2}=\varnothing$, and $t>0$. Then the join of $\left(X_{1}, d_{1}\right)$ and $\left(X_{2}, d_{2}\right)$ (relative to the parameter $t$ ) is the metric space $\left(X_{1} \cup X_{2}, d^{t}\right)$, where

$$
d^{t}(u, v)= \begin{cases}d_{1}^{t}(u, v) & \text { if } u, v \in X_{1} \\ d_{2}^{t}(u, v) & \text { if } u, v \in X_{2} \\ t & \text { if } u \in X_{i} \text { and } v \in X_{j}, \text { where } i \neq j\end{cases}
$$

As with graphs, $X_{1}+X_{2}$ always represents the metric space $\left(X_{1} \cup X_{2}, d^{t}\right)$, where in this case $t$ will be understood from the context.

We might hope that the metric dimension is additive with respect to the join, but unfortunately it is not. Let $X_{1}=\{1,3\}$ and $X_{2}=\{2,4\}$, each with the Euclidean metric, and let $t=1$. Then $X_{1} \cup X_{2}=\{1,2,3,4\}$ with the metric $d^{1}$, where $d^{1}(1,3)=d^{1}(2,4)=2$ and, for all other $x$ and $y, d^{1}(x, y)=1$. The bisectors in $X_{1}+X_{2}$ are $X_{1}, X_{2}$ and $\varnothing$, and from this we conclude that $\operatorname{dim}_{1}^{1}\left(X_{1}+X_{2}\right)=3$. Obviously, $\operatorname{dim}_{1}\left(X_{1}\right)=\operatorname{dim}_{1}\left(X_{2}\right)=1$, so that in this case, $\operatorname{dim}_{1}\left(X_{1}\right)+\operatorname{dim}_{1}\left(X_{2}\right)<\operatorname{dim}_{1}^{1}\left(X_{1}+X_{2}\right)$.

We now give some inequalities which hold for the join of two metric spaces. 
Theorem 8.2. Let $\left(X_{j}, d_{j}\right), j=1,2$, be metric spaces with $X_{1} \cap X_{2}=\varnothing$, and consider the join $\left(X_{1} \cup X_{2}, d^{t}\right)$. Then, for any positive integer $k$, we have

$$
\operatorname{dim}_{k}\left(X_{1}\right)+\operatorname{dim}_{k}\left(X_{2}\right) \leq \operatorname{dim}_{k}^{t}\left(X_{1}\right)+\operatorname{dim}_{k}^{t}\left(X_{2}\right) \leq \operatorname{dim}_{k}^{t}\left(X_{1}+X_{2}\right) .
$$

We shall now give an example which shows that (8.1) can hold; then we end with the proofs of Theorems 8.1 and 8.2, and stating a consequence of Theorem 8.2.

Example 8.3. Let $X=\mathbb{R}$ and $d(x, y)=|x-y|$, so that $\operatorname{dim}_{1}(X)=2$. We shall now show that if $t>0$ then $\operatorname{dim}_{1}^{t}(X)=+\infty$, so that (8.1) can hold. Suppose that $a<b$, and consider the bisector $B^{t}(a \mid b)$. If $x \leq a-2 t$, then $d^{t}(x, a)=d^{t}(x, b)=2 t$ so that $x \in B^{t}(a \mid b)$. Thus $B^{t}(a \mid b) \supset(-\infty, a-2 t]$. Now let $S$ be any finite set, and let $s$ be the largest element in $S$. Then $B^{t}(s+2 t, s+3 t) \supset(-\infty, s] \supset S$, so that $\operatorname{dim}_{1}^{t}(X)=+\infty$.

This is a convenient place to describe the notation that will be used in the following two proofs. We have metric spaces $\left(X_{1}, d_{1}\right)$ and $\left(X_{2}, d_{2}\right)$ with $X_{1} \cap X_{2}=\varnothing$. For $j=1,2$ we use $B_{j}(u \mid v)$ for the bisectors in $X_{j}$, and $\operatorname{dim}_{k}\left(X_{j}\right)$ for their metric dimensions. Now consider the join $\left(X_{1} \cup X_{2}, d^{t}\right)$, and its metric subspaces $\left(X_{j}, d^{t}\right)$. We use $B^{t}(u \mid v)$ and $B_{j}^{t}(u \mid v)$ for the bisectors in these spaces, and $\operatorname{dim}_{k}^{t}\left(X_{1}+X_{2}\right)$ and $\operatorname{dim}_{k}^{t}\left(X_{j}\right)$ for their metric dimensions. In general, we write $[B]^{c}$ for the complement of a bisector $B$ of any type.

We shall need the following lemma in our proof of Theorem 8.1.

Lemma 8.4. Let $(X, d)$ be a metric space, and suppose that $0<s<t$. Then $B(u \mid v) \subset$ $B^{t}(u \mid v) \subset B^{s}(u \mid v)$.

Proof. First, observe that for all real $r$, and all real, distinct $\alpha$ and $\beta$, we have $\min \{\alpha, r\}=$ $\min \{\beta, r\}$ if and only if (i) $r \leq \min \{\alpha, \beta\}$ or (ii) $\alpha=\beta$. Now suppose that $x \in B^{t}(u \mid v)$. Then $d^{t}(x, u)=d^{t}(x, v)$ so that $\min \{d(x, u), t\}=\min \{d(x, v), t\}$. This implies that $t \leq \min \{d(x, u), d(x, v)\}$ or $d(x, u)=d(x, v)$, and (since $s<t)$ in both cases we have $d^{s}(x, u)=d^{s}(x, v)$. Thus $B^{t}(u \mid v) \subset B^{s}(u \mid v)$. The proof that $B(u \mid v) \subset B^{t}(u \mid v)$ is trivial: if $x \in B(u \mid v)$ then $d(x, u)=d(x, v)$ so that $d^{t}(x, u)=d^{t}(x, v)$; hence $x \in$ $B^{t}(u \mid v)$.

The proof of Theorem 8.1. Let $A$ be any finite subset of $X$. Then, by Lemma 8.4, for all $u$ and $v$ in $X$ with $u \neq v$, we have

$$
\left|A \cap[B(u \mid v)]^{c}\right| \geq\left|A \cap\left[B^{t}(u \mid v)\right]^{c}\right| \geq\left|A \cap\left[B^{s}(u \mid v)\right]^{c}\right| .
$$

It follows that if $A$ is a $k$-metric generator for $\left(X, d^{s}\right)$ (that is, if, for all $u$ and $v, \mid A \cap$ $\left.\left[B^{s}(u \mid v)\right]^{c} \mid \geq k\right)$, then it is also a $k$-metric generator for $\left(X, d^{t}\right)$. Thus the minimum of $|S|$ taken over all $k$-metric generators $S$ of $\left(X, d^{t}\right)$ is less than or equal to the minimum over all $k$-metric generators of $\left(X, d^{s}\right)$; hence $\operatorname{dim}_{k}^{s}(X) \geq \operatorname{dim}_{k}^{t}(X)$. The proof that $\operatorname{dim}_{k}^{t}(X) \geq \operatorname{dim}_{k}(X)$ is entirely similar.

The proof of Theorem 8.2. The first inequality follows from Theorem 8.1. The inequality is trivially true if $\operatorname{dim}_{k}^{t}\left(X_{1}+X_{2}\right)=+\infty$, so we may assume that there is a $k$-metric basis, say $W$, of $X_{1}+X_{2}$. Thus $|W|=\operatorname{dim}_{k}^{t}\left(X_{1}+X_{2}\right)$. Now take any $u$ and $v$ in $X_{1}$; then

$$
B^{t}(u \mid v)=\left\{x \in X_{1} \cup X_{2}: d^{t}(x, u)=d^{t}(x, v)\right\}=B_{1}^{t}(u \mid v) \cup X_{2},
$$


so that, from Lemma 8.4, $\left[B^{t}(u \mid v)\right]^{c}=\left[B_{1}^{t}(u \mid v)\right]^{c} \subset X_{1}$. We put $W_{j}=W \cap X_{j}, j=1,2$. Then, if we let $u$ and $v$ vary over $X_{1}$, with $u \neq v$, we find that

$$
k \leq\left|\left[B^{t}(u \mid v)\right]^{c} \cap W\right|=\left|\left[B_{1}^{t}(u \mid v)\right]^{c} \cap X_{1} \cap W\right|=\left|\left[B_{1}^{t}(u \mid v)\right]^{c} \cap W_{1}\right|,
$$

so that $\operatorname{dim}_{k}^{t}\left(X_{1}\right) \leq\left|W_{1}\right|$. Similarly, $\operatorname{dim}_{k}^{t}\left(X_{2}\right) \leq\left|W_{2}\right|$, so that

$$
\operatorname{dim}_{k}^{t}\left(X_{1}\right)+\operatorname{dim}_{k}^{t}\left(X_{2}\right) \leq\left|W_{1}\right|+\left|W_{2}\right|=|W|=\operatorname{dim}_{k}^{t}\left(X_{1}+X_{2}\right)
$$

as required.

If $\left(X_{j}, d_{j}\right), j=1,2$, are metric spaces, each with diameter less than $t$, such that $X_{1} \cap X_{2}=\varnothing$, the for any $k$-metric basis $A_{i}$ of $\left(X_{j}, d_{j}\right), A_{1} \cup A_{2}$ is a $k$-metric generator for the join $\left(X_{1} \cup X_{2}, d^{t}\right)$. This shows that $\operatorname{dim}_{k}^{t}\left(X_{1}+X_{2}\right) \leq \operatorname{dim}_{k}\left(X_{1}\right)+\operatorname{dim}_{k}\left(X_{2}\right)$, and so Theorem 8.2 leads to the following corollary.

Corollary 8.5. Let $\left(X_{j}, d_{j}\right), j=1,2$, be metric spaces, each with diameter less than $t$, such that $X_{1} \cap X_{2}=\varnothing$. Then, for $k=1,2, \ldots$,

$$
\operatorname{dim}_{k}^{t}\left(X_{1}+X_{2}\right)=\operatorname{dim}_{k}\left(X_{1}\right)+\operatorname{dim}_{k}\left(X_{2}\right) .
$$

\section{References}

[1] S. Bau and A. F. Beardon, The metric dimension of metric spaces, Comput. Methods Funct. Theory 13 (2013), 295-305, doi:10.1007/s40315-013-0024-0.

[2] A. F. Beardon, The Geometry of Discrete Groups, volume 91 of Graduate Texts in Mathematics, Springer-Verlag, New York, 1983, doi:10.1007/978-1-4612-1146-4.

[3] M. Berger, Geometry I, Universitext, Springer-Verlag, New York, 1987, doi:10.1007/ 978-3-540-93815-6, translated from the French by M. Cole and S. Levy.

[4] L. M. Blumenthal, Theory and Applications of Distance Geometry, Clarendon Press, Oxford, 1953.

[5] L. M. Blumenthal, Theory and Applications of Distance Geometry, Chelsea Publishing, New York, 2nd edition, 1970.

[6] G. Chartrand, L. Eroh, M. A. Johnson and O. R. Oellermann, Resolvability in graphs and the metric dimension of a graph, Discrete Appl. Math. 105 (2000), 99-113, doi:10.1016/ s0166-218x(00)00198-0.

[7] A. Estrada-Moreno, Y. Ramírez-Cruz and J. A. Rodríguez-Velázquez, On the adjacency dimension of graphs, Appl. Anal. Discrete Math. 10 (2016), 102-127, doi:10.2298/aadm151109022e.

[8] A. Estrada-Moreno, J. A. Rodríguez-Velázquez and I. G. Yero, The $k$-metric dimension of a graph, Appl. Math. Inf. Sci. 9 (2015), 2829-2840, http: / / www . naturalspublishing . com/Article.asp?ArtcID=10013.

[9] A. Estrada-Moreno, I. G. Yero and J. A. Rodríguez-Velázquez, $k$-metric resolvability in graphs, in: J. A. Rodríguez-Velázquez and I. G. Yero (eds.), Jornadas de Matemática Discreta y Algorítmica, Elsevier, Amsterdam, volume 46 of Electronic Notes in Discrete Mathematics, 2014 pp. 121-128, doi:10.1016/j.endm.2014.08.017, papers from the 9th Conference held in Tarragona, July $7-9,2014$.

[10] A. Estrada-Moreno, I. G. Yero and J. A. Rodríguez-Velázquez, The $k$-metric dimension of corona product graphs, Bull. Malays. Math. Sci. Soc. 39 (2016), S135-S156, doi:10.1007/ s40840-015-0282-2. 
[11] A. Estrada-Moreno, I. G. Yero and J. A. Rodríguez-Velázquez, The $k$-metric dimension of the lexicographic product of graphs, Discrete Math. 339 (2016), 1924-1934, doi:10.1016/j.disc. 2015.12.024.

[12] F. Harary and R. A. Melter, On the metric dimension of a graph, Ars Combinatoria 2 (1976), 191-195.

[13] M. Heydarpour and S. Maghsoudi, The metric dimension of geometric spaces, Topology Appl. 178 (2014), 230-235, doi:10.1016/j.topol.2014.09.012.

[14] S. Khuller, B. Raghavachari and A. Rosenfeld, Landmarks in graphs, Discrete Appl. Math. 70 (1996), 217-229, doi:10.1016/0166-218x(95)00106-2.

[15] R. A. Melter and I. Tomescu, Metric bases in digital geometry, Comput. Vis. Graph. Image Process. 25 (1984), 113-121, doi:10.1016/0734-189x(84)90051-3.

[16] G. P. Murphy, A metric basis characterization of Euclidean space, Pacific J. Math. 60 (1975), 159-163, http://projecteuclid.org/euclid.pjm/1102868444.

[17] J. G. Ratcliffe, Foundations of Hyperbolic Manifolds, volume 149 of Graduate Texts in Mathematics, Springer, New York, 2nd edition, 2006.

[18] P. J. Slater, Leaves of trees, in: R. S. D. Thomas (ed.), Proceedings of the Sixth Southeastern Conference on Combinatorics, Graph Theory, and Computing, Utilitas Mathematica Publishing, Winnipeg, Manitoba, volume 14 of Congressus Numerantium, 1975 pp. 549-559, held at Florida Atlantic University, Boca Raton, Florida, February 17 - 20, 1975.

[19] I. G. Yero, A. Estrada-Moreno and J. A. Rodríguez-Velázquez, Computing the $k$-metric dimension of graphs, Appl. Math. Comput. 300 (2017), 60-69, doi:10.1016/j.amc.2016.12.005. 